\title{
Posición evolutiva de caracoles terrestres peruanos (Orthalicidae) entre los Stylommatophora (Mollusca: Gastropoda)
}

\section{Evolutionary position of Peruvian land snails (Orthalicidae) among Stylommatophora (Mollusca: Gastropoda)}

\author{
Jorge Ramirez ${ }^{1,2}$, Rina Ramírez ${ }^{1,2}$, Pedro Romero ${ }^{1,2}$, Ana Chumbe $^{1,2}$, Pablo Ramírez $^{3}$
}

1Laboratorio de Sistemática Molecular y Filogeografía, Facultad de Cien y Flogras Ciencias Blologicas, Universidad . Email Jorge Ramirez: jolobio@ hotmail.com

2Departamento de Malacología y Carcinología, Museo de Historia Carcinologla, Museo de Historia Mayor de San Marcos.

Av. Arenales 1256, Apartado 140434, Lima-14, Perú. Email Rina Ramírez: rina rm@yahoo.com

3Laboratorio de Microbiología Molecular, Facultad de Ciencias Biológicas, Universidad Nacional Mayor de San Marcos.

Presentado: 29/04/2009 Aceptado: 30/06/2009 Publicado online: 28/08/2009

\section{Resumen}

Los géneros Bostryx y Scutalus (Orthalicidae: Bulimulinae) son endémicos de América del Sur y están principalmente distribuidos en la vertiente occidental de los Andes del Perú. El objetivo del presente trabajo fue evaluar su posición evolutiva dentro de los gastrópodos Stylommatophora basada en el marcador mitocondrial 16S rRNA. Fueron obtenidas cuatro secuencias las que, junto con 28 de otros Stylommatophora disponibles en el GenBank, fueron alineadas con ClustalX. La reconstrucción filogenética se realizó mediante los métodos de Neighbor-Joining, Máxima Parsimonia, Máxima Verosimilitud e Inferencia Bayesiana. El alineamiento resultó en 371 sitios, con presencia de indels. Los dos géneros de la Familia Orthalicidae por primera vez incluidos en una filogenia molecular (Bostryx y Scutalus), formaron un grupo monofilético con otro miembro de la superfamilia Orthalicoidea (Placostylus), tal como lo obtenido con marcadores nucleares. Se discute también su relación evolutiva con otros caracoles terrestres.

Palabras claves: ADN mitocondrial, filogenia molecular, Orthalicoidea, Bostryx, Scutalus.

\section{Abstract}

The genera Bostryx and Scutalus (Orthalicidae: Bulimulinae) are endemics from South America. They are mainly distributed on the western slopes of the Peruvian Andes. The goal of the present work was to assess their evolutionary position among the stylommatophoran gastropods based on the 16S rRNA mitochondrial marker. Four sequences were obtained, and along with 28 sequences of other Stylommatophora retrieved from the GenBank, were aligned with ClustalX. The phylogenetic reconstruction was carried out using the methods of Neighbor-Joining, Maximum Parsimony, Maximum Likelihood and Bayesian inference. The multiple sequence alignment had 371 sites, with indels. The two genera of the family Orthalicidae for the first time included in a molecular phylogeny (Bostryx and Scutalus), formed a monophyletic group along with another member of the superfamily Orthalicoidea (Placostylus), result that is comparable with that obtained with nuclear markers. Their evolutionary relationship with other land snails is also discussed.

Keywords: Mitochondrial DNA, molecular phylogeny, Orthalicoidea, Bostryx, Scutalus.

\section{Introducción}

En la vertiente occidental de los Andes peruanos predominan las especies de caracoles terrestres de los géneros endémicos Bostryx y Scutalus (Orthalicoidea: Orthalicidae). La familia Orthalicidae se distribuye principalmente en la región Neotropical, con mayor diversidad en América del Sur (Breure, 1979). Otros géneros pertenecientes a la superfamilia Orthalicoidea habitan en Oceanía, como Placostylus, de la familia Placostylidae (Herbert \& Mitchell, 2009).

El ADN mitocondrial es un componente clave para desarrollar estudios filogenéticos debido a su alta tasa de mutación, herencia materna y falta de recombinación (Avise 2000, 2004). El gen 16S rRNA ha sido usado en varios análisis filogenéticos por su función y distribución universal, facilidad de aislar y caracterizar, así como por contener tanto regiones altamente variables como conservadas (Palumbi 1996). Podemos mencionar a Chiba (1999) quien estudió la evolución de los moluscos de las islas del Pacifico. Ross (1999) encontró una relación entre la distancia genética y la distribución geográfica de Discus macclintocki. Ramírez (2004) evaluó las relaciones evolutivas entre moluscos de la costa peruana (género Bostryx) y cómo los eventos El Niño/Oscilación Sur afectan sus poblaciones.
Usando la información proporcionada por un segmento variable del gen mitocondrial 16S rRNA se pretende evaluar la posición evolutiva de los géneros Bostryx y Scutalus dentro de los Stylommatophora, en una filogenia molecular junto con Placostylus, como grupo hermano, y representantes de diversos géneros de moluscos terrestres pulmonados.

\section{Materiales y métodos}

Tres especies de caracoles terrestres endémicos del Perú fueron capturados en tres ecosistemas de lomas (Tabla 1):

Phylum Mollusca

Clase Gastropoda

Subclase Pulmonata

Orden Eupulmonata

Suborden Stylommatophora

Infraorden Sigmurethra

Familia Orthalicidae

$$
\begin{aligned}
& \text { Bostryx sordidus (Fig. 1) } \\
& \text { Bostryx scalariformis (Fig. 2) } \\
& \text { Scutalus versicolor (Fig. 3) }
\end{aligned}
$$

Los especímenes de referencia fueron depositados en la colección científica del Departamento de Malacología y Carcinología

\begin{tabular}{|c|c|c|}
\hline Especie & Localidad en Perú & \# Accesión \\
\hline Bostryx scalariformis (Broderip, 1832) & Dept. Lima: Cerro de Agua, $11^{\circ} 22^{\prime} 17,5^{\prime \prime} \mathrm{S} ; 77^{\circ} 26^{\prime} 22,6^{\prime \prime} \mathrm{W}$ & FJ969796 \\
\hline Bostryx sordidus (Lesson, 1826) & Dept. Ancash: Lomas de Lupín, $10^{\circ} 24^{\prime} 79^{\prime \prime}$ S; $77^{\circ} 54^{\prime} 86^{\prime \prime} \mathrm{W}$ & FJ969797 \\
\hline Scutalus versicolor (Broderip, 1832) & Dept. Ancash: Lomas de Mongón, $09^{\circ} 37^{\prime} 57,9^{\prime \prime} \mathrm{S} ; 78^{\circ} 16^{\prime} 41,5^{\prime \prime} \mathrm{W}$ & FJ969798 FJ969799 \\
\hline
\end{tabular}

Tabla 1. Especies de caracoles terrestres de la Familia Orthalicidae consideradas en el presente estudio. Se indican la procedencia de las muestras así como el número de accesión de las secuencias del marcador mitocondrial 16S rRNA, depositadas en el GenBank. 


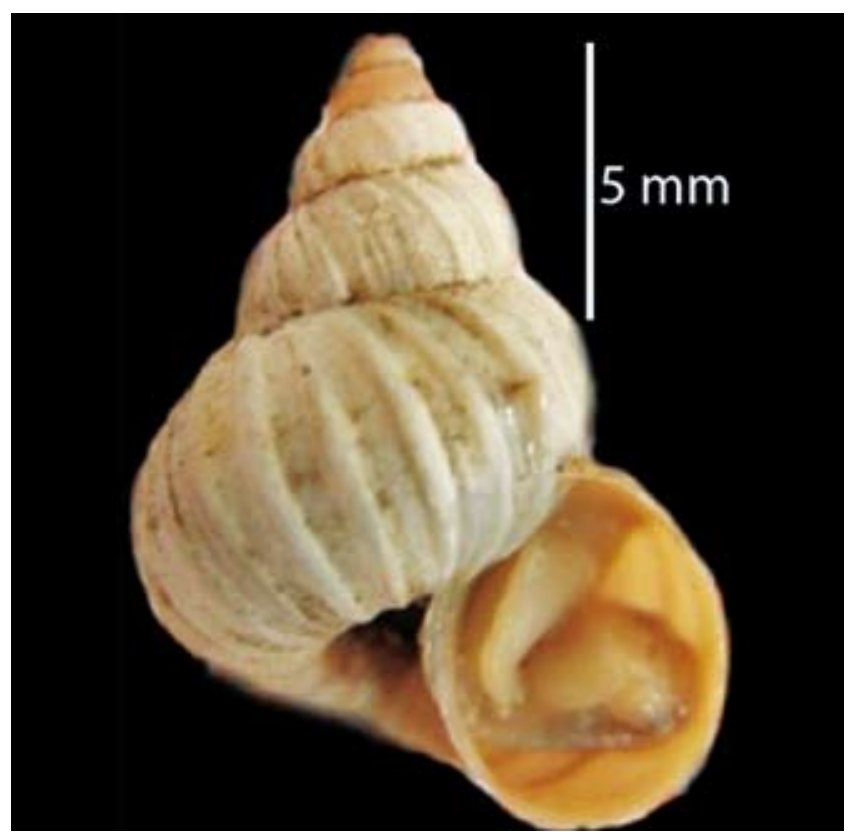

Figura 1. Bostryx scalariformis

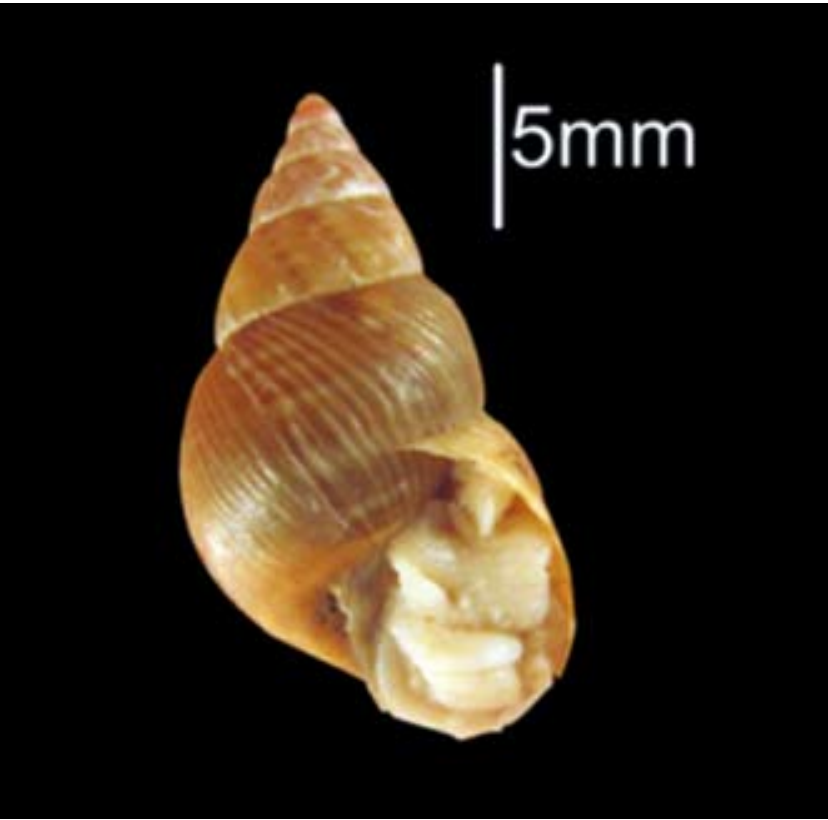

Figura 2. Bostryx sordidus

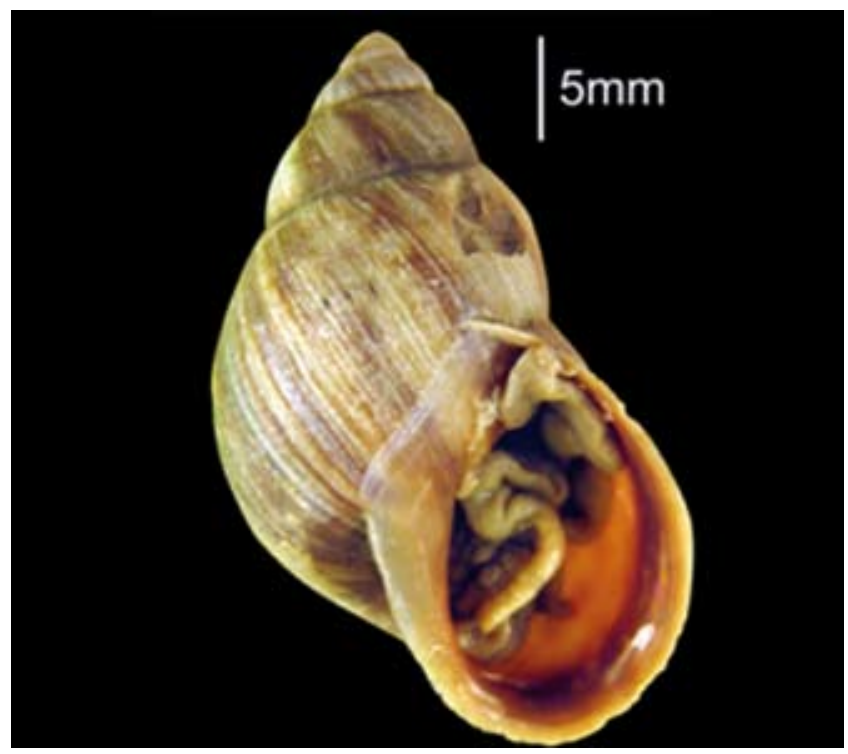

Figura 3. Scutalus versicolor del Museo de Historia Natural de la Universidad Nacional Mayor de San Marcos. El DNA total fue aislado de músculo del pie usando una modificación del método de CTAB (Doyle y Doyle 1987). Un segmento variable del gen mitocondrial $16 S$ rRNA fue amplificado por PCR usando primers y protocolos descritos por Ramírez (2004). Ambas hebras de los amplificados fueron secuenciadas usando los servicios de Macrogen Inc. USA. La edición manual de las secuencias fue realizada usando Chromas (McCarthy 1996); el ensamblaje de las secuencias consenso fue llevado a cabo mediante Cap3win (Huang y Madan 1999). Las secuencias fueron depositadas en el GenBank (Números de Accesión: FJ969796 - FJ969799).

\section{Alineamiento múltiple de secuencias e inferencia filogenética}

Las cuatro secuencias obtenidas, dos de Bostryx spp. y dos de Scutalus versicolor, fueron alineadas junto con otras 26 secuencias de Stylommatophora y dos de pulmonados no-Stylommatophora obtenidas en el GenBank (Tabla 2). De todas las secuencias del gen $16 \mathrm{~S}$ rRNA disponibles, fueron seleccionados taxa representantes de caracoles pulmonados terrestres del suborden Stylommatophora (Wade et al. 2006); se encontró sólo una secuencia de la superfamilia Orthalicoidea, correspondiente a Placostylus (Placostylidae); las secuencias seleccionadas pertenecen tan solo al clado "no-achatinoideo" pues no se encontraban disponibles representantes del clado "achatinodeo". Se usó a los dos pulmonados no-estilommatóforos (Carychium minimum y Siphonaria pectinata) como grupo externo. Originalmente fue usada sólo una secuencia por género de gastrópodo estilommatóforo, pero al observarse la inconsistencia de las posiciones de Succinea, Mastus, Discus, Helix y Albinaria, se procedió a agregar una y luego otra secuencia más, para corregir el efecto de "atracción de la rama larga" (long branch atraction) (Swofford et al. 1996).

El alineamiento múltiple de secuencias, llevado a cabo usando ClustalX 2.09 (Larkin 2007), fue corregido manualmente con la ayuda de Bioedit 6.0 (Hall 1999). El modelo de substitución nucleotídica fue estimado mediante los programas Modeltest 3.7 (Posada y Crandall 1998) y MrModeltest 2.3 (Nylander 2004). La reconstrucción filogenética fue llevada a cabo con el uso del programa MEGA 4 (Tamura et al. 2007) para el método Neighbor-Joining (NJ), PAUP* 4.0b10 (Swofford 1998) para los métodos, Máxima Parsimonia (MP) y Máxima Verosimilitud (ML), y el programa Mr. Bayes 3.1.2 (Huelsenbeck y Ronquist 2001) para el método de reconstrucción filogenética por Inferencia Bayesiana (IB).

Para el análisis filogenético con el algoritmo de NeighborJoining, fue usado el modelo de Maximum Composite Likelihood. Para los análisis con Máxima Parsimonia se empleó un algoritmo de búsqueda heurístico obteniéndose el árbol inicial por adición secuencial (stepwise addition), utilizando el algoritmo de Bisección-reconexión de árboles (Tree-Bisection-Reconnection, TBR) para el intercambio de ramas (Branch-swapping). En ambos métodos fueron construidos árboles filogenéticos tanto sin considerar gaps, como considerándolos. Para el análisis de Máxima Verosimilitud en PAUP* se realizó una búsqueda heurística obteniéndose el árbol inicial por adición secuencial para luego realizar la adición de las secuencias por AsIs con cinco repeticiones; el algoritmo de TBR fue usado para el reajuste de topología (Branch-swapping). Para el análisis Bayesiano se usaron cuatro cadenas de un algoritmo de Cadena de Markov-Monte 
Tabla 2. Especies, referencias y número de accesión de las secuencias del gen 16S rRNA obtenidas del GenBank.

\begin{tabular}{|c|c|c|c|}
\hline Familia & Especie & Referencia & \# Accesión \\
\hline \multicolumn{4}{|c|}{ Pulmonados Stylommatophora (Phylum Mollusca, Clase Gastropoda, Subclase Pulmonata, Orden Eupulmonata, Suborden Stylommatophora) } \\
\hline \multicolumn{4}{|c|}{ Infraorden Orthurethra } \\
\hline \multirow[t]{2}{*}{ Chondrinidae } & Chondrina avenacea (Bruguière, 1792) & Ketmaier et al., 2006 & DQ305071 \\
\hline & Solatopupa cianensis (Caziot, 1910) & Ketmaier et al., 2006 & DQ305070 \\
\hline \multirow[t]{3}{*}{ Enidae } & Mastus cretensis (Pfeiffer, 1846) & Parmakelis \& Mylonas, 2004 & AY485926 \\
\hline & Mastus procax/hemmeni Maassen, 1995 & Parmakelis \& Mylonas, 2004 & AY485930 \\
\hline & Mastus carneolus (Mousson 1863) & Parmakelis \& Mylonas, 2004 & AY485928 \\
\hline \multicolumn{4}{|c|}{ Infraorden Elasmognatha } \\
\hline \multirow[t]{3}{*}{ Succineidae } & Succinea caduca Mighels, 1845 & Holland \& Cowie, 2007 & EF217262 \\
\hline & Succinea caduca Mighels, 1845 & Holland \& Cowie, 2007 & EF217304 \\
\hline & Succinea caduca Mighels, 1845 & Holland \& Cowie, 2007 & EF217264 \\
\hline \multicolumn{4}{|c|}{ Infraorden Sigmurethra } \\
\hline \multirow[t]{3}{*}{ Clausiliidae } & Albinaria discolor (Pfeiffer, 1846) & Douris et al., 2007 & DQ665354 \\
\hline & Albinaria brevicollis (Pfeiffer, 1850) & Douris et al., 2007 & DQ665349 \\
\hline & Albinaria turrita (Pfeiffer, 1850) & Douris et al., 2007 & DQ665362 \\
\hline \multirow[t]{2}{*}{ Bradybaenidae } & Ainohelix editha (Adams, 1868) & Teshima et al., 2003 & AY137577 \\
\hline & Acusta despecta (Sowerby, 1839) & Teshima et al., 2003 & AY137578 \\
\hline \multirow[t]{6}{*}{ Polygyridae } & Lobosculum pustulosa & Perez, 2004 & DQ086017 \\
\hline & Triodopsis vannostrandi (Bland, 1875) & Perez, 2004 & DQ085935 \\
\hline & Millerelix mooreana (Binney, 1858) & Perez, 2004 & DQ085999 \\
\hline & Praticolella sp. & Perez, 2004 & DQ098157 \\
\hline & Polygyra cereolus (Mühlfeld, 1816) & Perez, 2004 & DQ086001 \\
\hline & Daedalochila hippocrepis (Pfeiffer, 1848) & Perez, 2004 & DQ086005 \\
\hline \multirow[t]{3}{*}{ Endodontidae } & Discus macclintocki (Baker, 1928) & Ross, 1999 & AF064438 \\
\hline & Discus macclintocki (Baker, 1928) & Ross, 1999 & AF064437 \\
\hline & Discus macclintocki (Baker, 1928) & Ross, 1999 & AF064436 \\
\hline \multirow[t]{3}{*}{ Helicidae } & Helix aspersa maxima (Taylor 1883) & Guiller et al., 2001 & AF126143 \\
\hline & Helix aspersa Muller, 1774 & Abdulmawjood \& Buelte, 2001 & AF434797 \\
\hline & Helix pomatia Linnaeus, 1758 & Manganelli et al., 2005 & AY741411 \\
\hline Placostylidae & Placostylus bivaricosus (Gaskon, 1855) & Ponder et al., 2003 & AY165847 \\
\hline \multicolumn{4}{|c|}{ Pulmonado No-Stylommatophora (Phylum Mollusca, Clase Gastropoda, Subclase Pulmonata, Orden Eupulmonata) } \\
\hline Carychiidae & Carychium minimum Muller, 1774 & Klussmann-Kolb et al., 2008 & EF489308 \\
\hline \multicolumn{4}{|c|}{ Pulmonado No-Stylommatophora (Phylum Mollusca, Clase Gastropoda, Subclase Pulmonata, Orden Basommatophora) } \\
\hline Siphonariidae & Siphonaria pectinata (Linnaeus, 1758) & Okusu et al., 2003 & AY377627 \\
\hline
\end{tabular}

Carlo, por 25 millones de generaciones, muestreando cada 2500 generaciones. Un árbol consenso fue construido usando los últimos 1000 árboles (burn-in= 9001 muestras).

El soporte estadístico de los nodos de los árboles filogenéticos encontrados fue evaluado usando bootstrap, con 1000 remuestreos para el caso de $\mathrm{NJ}$ y $\mathrm{MP}$, mientras que para el caso de Máxima Verosimilitud fueron 100. En el caso del análisis bayesiano, el soporte estadístico de los nodos fue proporcionado por las probabilidades posteriores.

A fin de comparar las topologías de los árboles obtenidos con los distintos métodos filogenéticos usamos las pruebas de Kishino-Hasegawa (KH) (Kishino y Hasegawa 1989) y Shimodaira-Hasegawa (SH) (Shimodaira y Hasegawa 1999), en PAUP*. Se usó la opción "fullopt" para generar una prueba de distribución por el remuestreo del método de log-likelihood estimado (Kishino et al. 1990). Los valores de log-likelihood fueron estimados usando un modelo totalmente optimizado con un valor de boostrap de 1000.

\section{Resultados}

Los cuatro amplificados del segmento del gen mitocondrial 16S rRNA para las especies de Bostryx y Scutalus de la región costera del Perú resultaron entre 328 y 331 pb. El alineamiento de ellas, junto con otras 26 secuencias del clado "no-achatinoideo" del Suborden Stylommatophora (Tabla 2) y de Carychium y
Siphonaria, resultó en 371 sitios con presencia de indels; 223 sitios fueron filogenéticamente informativos. El mejor modelo de substitución nucleotídica para los datos fue el GTR+I+G con 0,9647 como parámetro alfa de la distribución gamma y 0,2712 como la proporción de sitios invariantes. Las frecuencias nucleotídicas fueron $\mathrm{T}=33,1, \mathrm{C}=12,2, \mathrm{~A}=36,0$ y $\mathrm{G}=18,7$. La elección del mejor modelo y sus parámetros con el escogido por el programa Modeltest 3.7 fue coincidente con los hallados por el MrModeltest 2.3.

En todos los árboles encontrados, usando diversos métodos filogenéticos (NJ, MP, ML e IB), los géneros Bostryx y Scutalus (Familia Orthalicidae) quedaron más cercanamente relacionados entre sí y formaron un grupo monofilético con el género Placostylus (Placostylidae), conformando la Superfamilia Orthalicoidea, con valores de bootstrap mayores a 90\% (Fig. 4 y 5).

El clado Orthalicoidea quedó en posición basal entre los gastrópodos estilommatóforos, en las topologías de NJ (Fig. 4A) y ML (Fig. 5B); sin embargo, los otros clados tienen muy bajo soporte estadístico, por lo que la posición de Orthalicoidea sería incierta. Caso similar sucede en las topologías encontradas con MP (Fig. 4B) e IB (Fig. 5A). Al realizar la comparación de todas las topologías por las pruebas de $\mathrm{KH}$ y $\mathrm{SH}$ se obtuvo como la más correcta a la obtenida por el método filogenético de Máxima Verosimilitud, seguida por la obtenida mediante Neighbor-Joining. 


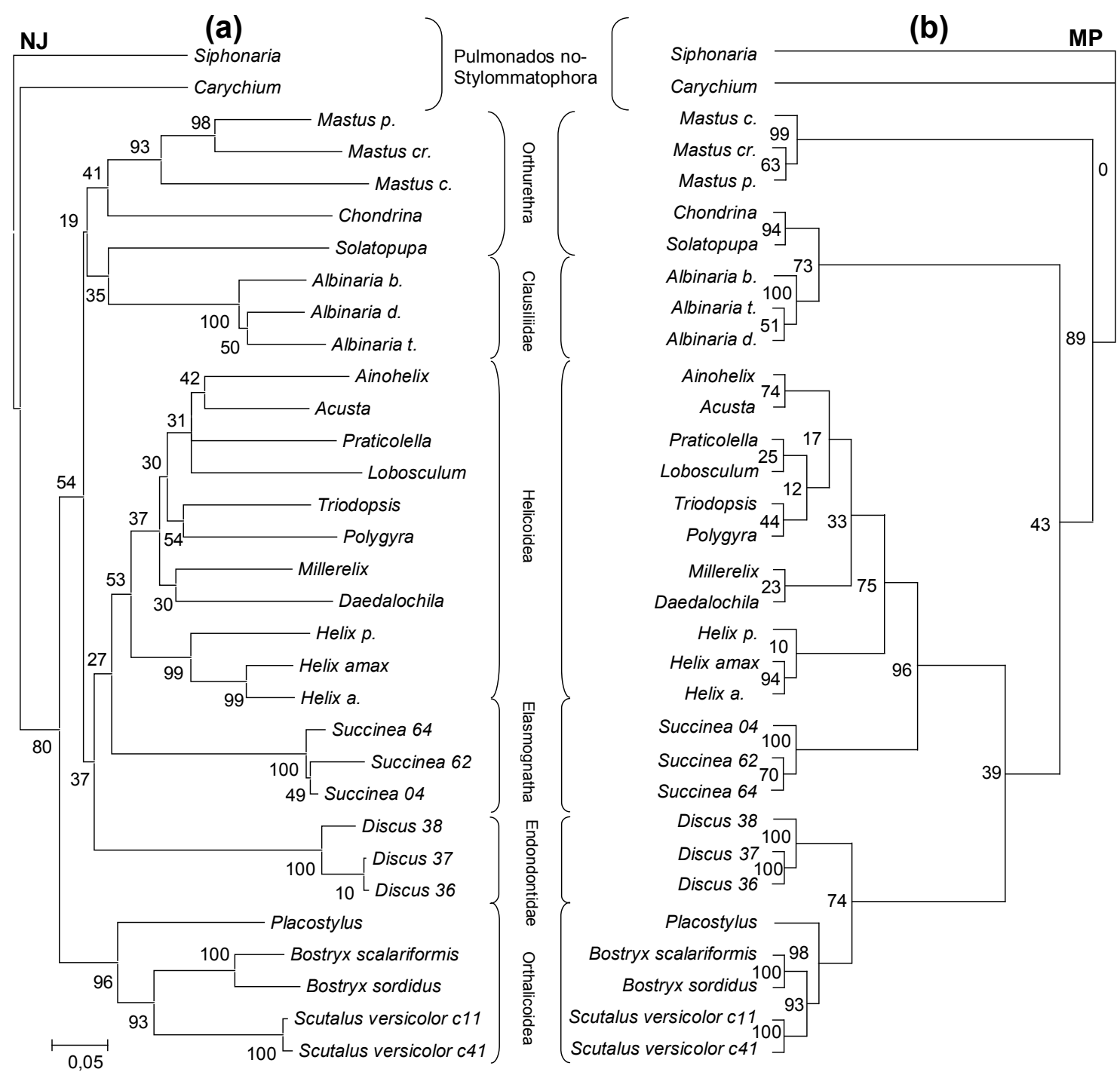

Figura 4. Árboles filogenéticos para el marcador mitocondrial 16S rRNA. (a): Topología obtenida usando NJ considerando gaps. (b): Topología obtenida usando MP considerando gaps como "nuevo estado". Los números corresponden a valores de bootstrap. La escala corresponde al número de substituciones por sitio. Note los altos valores de bootstrap para el grupo monofilético conformado por Bostryx y Scutalus (Familia Orthalicidae), dentro del clado de la superfamilia Orthalicoidea.

\section{Discusión}

El presente estudio incluyó a dos géneros principales de la Familia Orthalicidae (Bostryx y Scutalus) en una filogenia basada en el marcador mitocondrial $16 \mathrm{~S}$ rRNA, que contuvo 30 secuencias de representantes del clado "no-achatinoideo" (Wade et al. 2001, 2006) de gastrópodos terrestres del Suborden Stylommatophora, y a dos pulmonados no-Stylommatophora como grupo externo. Estudios previos, usando el mismo marcador mitocondrial, en los gastrópodos Euthyneura, encontraron a los gastrópodos Pulmonados (Amerianna carinata, Lymnaea stagnalis, Albinaria turrita y Cepaea nemoralis) formando un clado, aunque con bajo valor de bootstrap (<60\%) (Thollesson 1999). Grande et al. (2004), al usar los genes 16S rRNA y COI en una filogenia de la Clase Gastropoda, incluyendo cinco especies de Stylommatophora (Rumina decollata, Elona quimperiana, Helix aspersa, Cepaea nemoralis y Albinaria coerulea), encontraron que Pulmonata no era un grupo monofilético, pero que los pulmonados terrestres estilommatóforos sí tenían una naturaleza monofilética. Recientemente, este último resultado ha sido corroborado usando genes nucleares (18S rRNA y 28S rRNA) y mitocondriales (16S rRNA y COI) (Klussmann-Kolb 2008). Wade et al. (2001; 2006), usando marcadores nucleares (extremo $3^{\prime}$ del gen $5.8 \mathrm{~S}$
rRNA, ITS-2, y el extremo 5' del gen 28S rRNA) para el análisis filogenético de gastrópodos Pulmonados, encontraron al grupo monofilético Stylommatophora formando dos clados, que nominaron como el clado "no-achatinoideo", donde se agruparon la mayoría de familias, y el clado "achatinoideo". Los géneros de la Superfamilia Orthalicoidea utilizados por ellos [Placostylus (Placostylidae), Bulimulus y Drymaeus (Orthalicidae) y Gaeotis (Amphibulimidae)] formaron un grupo monofilético dentro del clado "no-achatinoideo". En el presente estudio, usando el marcador mitocondrial 16S rRNA, los dos géneros de la Familia Orthalicidae por primera vez incluidos en una filogenia molecular (Bostryx y Scutalus), formaron un grupo monofilético con Placostylus. Asimismo, entre los Stylommatophora "noachatinoideos" usados en los análisis se formó también el clado Helicoidea, aunque con bajo sustento estadístico. Por otro lado, si bien las secuencias de Albinaria (Familia Clausiliidae) formaron un clado independiente en la topología de MP, en los árboles filogenéticos obtenidos por NJ, IB y ML quedó junto con los Orthurethra; ello puede deberse a que la filogenia del presente estudio no incluye representantes de los Arionoidea ni Limacoidea, que se posicionan entre los clados de los Orthurethra y Clausiliodea en la filogenia nuclear (Wade et al., 2006). 


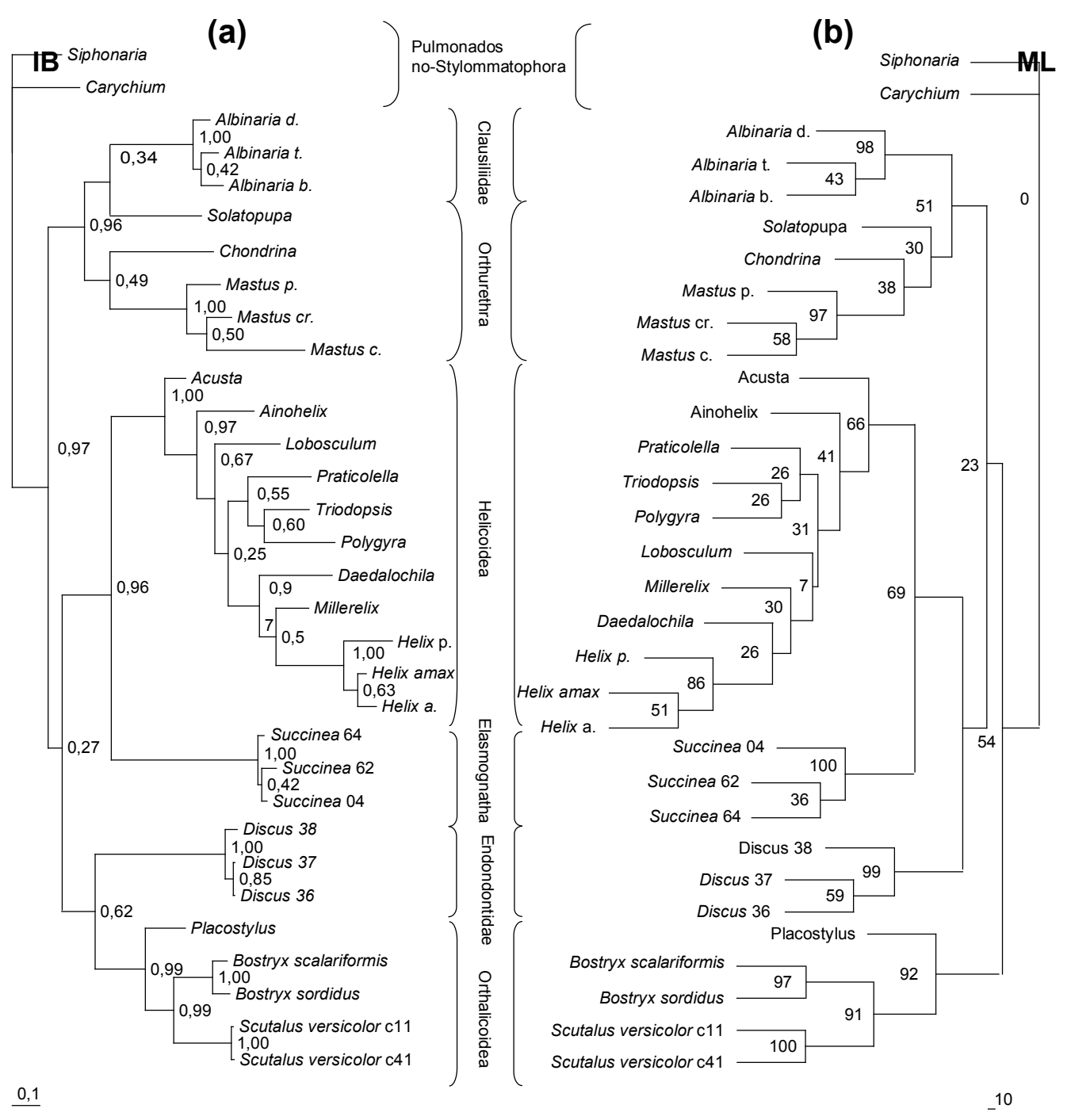

Figura 5. Árboles filogenéticos obtenidos usando IB (a) y ML (b), para el marcador mitocondrial 16S rRNA. Los números corresponden a valores de probabilidades posteriores bayesianas (a) y a bootstrap (b). La escala corresponde al número de substituciones por sitio. Note los altos valores para el grupo monofilético conformado por la Superfamilia Orthalicoidea.

Sobre la base de marcadores nucleares ha sido demostrado que los caracoles anfibios del género Succinea (Elasmognatha) forman parte de los pulmonados estilommatóforos; Tillier et al. (1996) usaron el gen $28 \mathrm{~S}$ rRNA y Dutra-Clarke et al. (2001) el gen $18 \mathrm{~S}$ rRNA. Wade et al. (2006) encontraron que los Elasmognatha Succinea y Athoracophorus forman un grupo monofilético bien soportado dentro del clado "no-achatinoideo". En el presente estudio también es soportada la posición de Succinea dentro del clado de moluscos pulmonados estilommatóforos; sin embargo, usando el marcador mitocondrial $16 \mathrm{~S}$ rRNA, la posición de la Familia Succineidae difiere de la nuclear en que no está cercanamente relacionada a Orthalicoidea sino más bien a Helicoidea. Ello se debería al hecho de no estar presente Athoracophorus en el análisis, ambos del Infraorden Elasmognatha, lo que estaría haciendo que quede bajo el efecto de "atracción de la rama larga", lo que significa que su posición varía debido a que sus secuencias son tremendamente divergentes en relación a las otras usadas en el análisis filogenético (Swofford et al. 1996).

Las relaciones evolutivas dentro de los distintos grupos de Stylommatophora aun permanecen inciertas, debido al alto nivel de homoplasia que presenta este grupo, probablemente debido a una radiación explosiva en tiempos recientes (Tillier et al. 1996).
Usando la información proporcionada por las secuencias inéditas de 16S rRNA de Bostryx y Scutalus, en este trabajo se corrobora la naturaleza monofilética de la Familia Orthalicidae y la Superfamilia Orthalicoidea, como parte del clado "noachatinoideo" de los gastrópodos terrestres del Suborden Stylommatophora, así como la eficacia del marcador mitocondrial $16 \mathrm{~S}$ rRNA para resolver relaciones evolutivas profundas.

\section{Agradecimientos}

El presente trabajo es parte de proyectos del Instituto de Investigación en Ciencias Biológicas "Antonio Raimondi" (ICBAR) de la Facultad de Ciencias Biológicas de la Universidad Nacional Mayor de San Marcos (Códs. 051001071 y 061001071). Estuvo financiado por la UNMSM, mediante el Vicerrectorado de Investigación y su Consejo Superior de Investigación.

\section{Literatura Citada}

Abdulmawjood A. \& M. Bülte. 2001. Snail Species Identification by RFLP-PCR and Designing of Species-Specific Oligonucletid Primers. Journal of Food Science 66 (9): 1287-1293.

Avise J.C. 2000. Phylogeography: the history and formation of species. Cambridge, MA: Harvard University Press.

Avise J.C. 2004. Molecular markers, natural history and evolution. 2nd edn. Sunderland, MA: Sinauer Associates. 
Breure A. 1979. Systematics, phylogeny and zoogeography of Bulimulidae (Mollusca). Zoologische Verhandelingen 168: 1-215.

Chiba S. 1999. Accelerated evolution of land snails Mandarina in the oceanic Bonin Islands. Evolution 53: 460-471.

Douris V., S. Giokas, D. Thomaz, R. Lecanidou, \& G. Rodakis. 2007. Inference of evolutionary patterns of the land snail Albinaria in the Aegean archipelago: Is vicariance enough?. Molecular Phylogenetics and Evolution 44 (3): 1224-1236.

Doyle J.J. \& J.L. Doyle. 1987. A rapid DNA isolation procedure for small amounts of fresh leaf tissue. Phytochemical Bulletin 19: 11-15.

Dutra-Clarke A., C. Williams, R. Dickstein, N. Kaufer \& J. Spolita. 2001. Inferences on the phylogenetics relationships of Succineidae (Mollusca, Pulmonata) based on the $18 \mathrm{~S}$ rRNA gene. Malacologia 43: 223-236

Grande C., J. Templado, J. Cervera \& R. Zardoya. 2004. Molecular Phylogeny of Euthyneura (Mollusca: Gastropoda). Molecular Biology and Evolution 21(2): 303-313.

Guiller A., M. Coutellec-Vreto, L. Madec, \& J. Deunff. 2001. Evolutionary history of the land snail Helix aspersa in the Western Mediterranean: preliminary results inferred from mitochondrial DNA sequences. Molecular Ecology 10 (1): 81-87.

Hall T.A. 1999. BioEdit: a user-friendly biological sequence alignment editor and analysis program for Windows 95/98/NT. Nucleic Acids Symposium Series 41: 95-98.

Herbert D \& A. Mitchell. 2009. Phylogenetic relationships of the enigmatic land snail genus Prestonella - the missing African element in the Gondwanan superfamily Orthalicoidea (Mollusca: Stylommatophora). Biological Journal of the Linnean Society Lond 96: 203-221.

Holland B. \& R. Cowie. 2007. A geographic mosaic of passive dispersal: population structure in the endemic Hawaiian amber snail Succinea caduca (Mighels, 1845). Molecular Ecology 16 (12): 2422-2435.

Huang X. \& A. Madan. 1999. CAP3: A DNA Sequence Assembly Program. Genome Research 9: 868-877.

Huelsenbeck J. \& F. Ronquist. 2001. MRBAYES: Bayesian inference of phylogenetic trees. Bioinformatics 17(8): 754-755

Ketmaier V., F. Giusti, \& A. Caccone. 2006. Molecular phylogeny and historical biogeography of the land snail genus Solatopupa (Pulmonata) in the peri-Tyrrhenian area. Molecular Phylogenetics and Evolution 39 (2): 439-451.

Kishino H. \& M. Hasegawa. 1989. Evaluation of the maximum likelihood estimate of the evolutionary tree topologies from DNA sequence data, and the branching order in Hominoidea. Journal of Molecular Evolution 29: 170-179.

Kishino H., T. Miyata \& M. Hasegawa. 1990. Maximum likelihood inference of protein phylogeny and the origin of chloroplasts. Journal of Molecular Evolution 30: 151-160.

Klussmann-Kolb A., A. Dinapoli1, K. Kuhn, B. Streit \& C. Albrecht. 2008. From sea to land and beyond - New insights into the evolution of euthyneuran Gastropoda (Mollusca). BMC Evolutionary Biology 8:57.

Larkin M., G. Blackshields, N. Brown, R. Chenna, P. McGettigan, H. McWilliam, F. Valentin, I. Wallace, A. Wilm, R. Lopez, J. Thompson, T. Gibson \& D. Higgins. 2007. Clustal W and Clustal X version 2.0. Bioinformatics 23: 2947-2948.

Manganelli G., N. Salomone, \& F. Giusti. 2005. A molecular approach to the phylogenetic relationships of the western palaearctic Helicoidea (Gastropoda: Stylommatophora). Biological Journal of the Linnean Society. 85 (4): 501 512 .
McCarthy C. 1996. Chromas: version 1.3. Griffith University, Brisbane, Australia.

Nylander J. 2004. MrModeltest 2.3. Program distributed by the author. Evolutionary Biology Centre, Uppsala University.

Okusu A., E. Schwabe, D.J. Eernisse \& G. Giribet. 2003. Towards a phylogeny of chitons (Mollusca, Polyplacophora) based on combined analysis of five molecular loci. Organisms Diversity \& Evolution 3 (4): 281-302.

Palumbi R. 1996. Nucleic Acids II: The polymerase Chain Reaction. In: Hillis, D., C. Moritz \& B. Mable, eds.: Molecular Systematics. pp. 205-247.

Parmakelis A. \& M. Mylonas. 2004. Dispersal and population structure of two sympatric species of the Mediterannean land snail genus Mastus (Gastropoda, Pulmonata, Enidae). Biological Journal of the Linnean Society. 83: 131-144.

Perez K. 2004. Systematic Relationships within the Genus Praticolella (Gastropoda: Pulmonata: Polygyridae) from the Southern United States \& Mexico. Centenary Research Grant Report, Bulletin of the Malacological Society of London 42: 5.

Ponder W., D. Colgan, D. Gleeson, \& G. Sherley. 2003. The relationships of Placostylus from Lord Howe Island. Molluscan Research 23: 159-178.

Posada D. \& K. Crandall 1998. Modeltest: testing the model of DNA substitution. Bioinformatics 14(9): 817-818.

Ramírez R. 2004. Sistemática e Filogeografia dos Moluscos do Ecossistema de "Lomas" do Deserto da Costa Central do Peru. Tese de Doutorado em Zoologia. Faculdade de Biociências, PUCRS, Porto Alegre, Brasil.

Ross T. 1999. Phylogeography and conservation genetics of the Iowa Pleistocene snail. Molecular Ecology 8(9): 1363-1373.

Shimodaira H. \& M. Hasegawa. 1999. Multiple Comparisons of LogLikelihoods with Applications to Phylogenetic Inference. Molecular Biology and Evolution 16: 1114-1116.

Swofford D. 1998. PAUP*: Phylogenetic analysis using parsimony (*and other methods). Version 4.0b8a. Sunderland, MA: Sinauer.

Swofford D., G.J. Olsen, P.J. Waddell \& D.M. Hillis. 1996. Phylogenetic inference. In: D.M. Hillis, C. Moritz and B.K. Mable, eds. Molecular Systematics. 2nd edn. Sunderland, MA: Sinauer Associates. Pp. 407-514.

Tamura K., J. Dudley, M. Nei \& S. Kumar. 2007. MEGA4: Molecular Evolutionary Genetics Analysis (MEGA) software version 4.0. Molecular Biology and Evolution 24: 1596-1599.

Teshima H., A. Davison, Y. Kuwahara, J. Yokoyama, S. Chiba, T. Fukuda, H. Ogimura, \& M. Kawata. 2003. The evolution of extreme shell shape variation in the land snail Ainohelix editha: a phylogeny and hybrid zone analysis. Molecular Ecology 12 (7): 1869-1878

Thollesson M. 1999. Phylogenetic analysis of Euthyneura (Gastropoda) by means of the $16 \mathrm{~S}$ rRNA gene: use of a 'fast' gene for 'higher-level' phylogenies. Proceedings of the Royal Society of London Series B 266: 75-83.

Tillier S., M. Masselot \& A. Tillier. 1996. Phylogenetic relationships of the pulmonate gastropods from rRNA sequences, and tempo and age of the stylommatophoran radiation. In: Taylor, J.D., ed.: Origin and evolutionary radiation of the Mollusca. Oxford: Oxford University Press, 267-284.

Wade C., P. Mordan \& B. Clarke. 2001. A phylogeny of the land snails (Gastropoda: Pulmonata). Proceedings of the Royal Society of London Series B 268: 413-422.

Wade C., P. Mordan, \& F. Naggs. 2006. Evolutionary relationships among the Pulmonate land snails and slugs (Pulmonata, Stylommatophora). Biological Journal of the Linnean Society 87(4): 593-610. 